hemodynamically stabile and it was decided to discontinue sedation in order to evaluate brain function. 24 hours after the complete cessation of sedation there was no neurological improvement indicating she was probably brain dead. Two neurological examinations were done with observation period of 12 hours, including apnoea test, demonstrating absent cerebral and brainstem function and confirming the diagnosis of brain death. Croatian legal regulations mandate the use of confirmatory tests to supplement the clinical examination so we decided to do scintigraphic blood flow study, ancillary test of choice in our institution. First test failed to show 'hollow skull phenomenon', as the second one done 24 hours later. Because of craniotomy, it was impossible to consider the use of electroencephalography and there was not enough experience and expertise with somatosensory evoked potentials, leaving as with only blood flow studies. Since experts in their field did not expect different results with cerebral angiography or Transcranial Doppler, we repeated cerebral perfusion scintigraphy four more times in the next forty days without confirming the clinical diagnosis of brain death.

During that time there was no change in neurological status. We were able to maintain vital functions for two months in total after which the child died.

Our experience shows that even though neurological examinations demonstrated absent cerebral and brainstem function blood flow study failed to confirm the diagnosis of brain death, probably because of breached cranial vault, in this case because of craniotomy.

\section{THE CASE OF GROWTH ARREST WHICH APPEARED TO BE HYPOTHYROIDISM AND PITUATRY HYPERPLASIA}

Iryna Artcukevich*, O Zenevich, V Zhemoytiak, L Collins. Children Regional Hospial Grodno Belarus

\subsection{6/archdischild-2021-europaediatrics.497}

Background Normal growth is the main of all fundamental characteristics of childhood. Deviation from a normal pattern of growth plate is dependent on variety of different factors, including nonendocrine disorders and hormonal stimuli: growth hormone, IGF-I, sex steroids, thyroid hormones, paracrine growth factors, and cytokines.

Case We present 12 years old girl who was referred to endocrinology department (she had reduced of the height velocity in the last 3 years old and appeared overweight. The girl was born by the first normal pregnancy.

Her birth weight was $3.8 \mathrm{~kg}$, length $54 \mathrm{~cm}$. Her intellectual development and school performance was good. She did not have any complains. Her weight was $32 \mathrm{~kg}$ (10-25 cen-

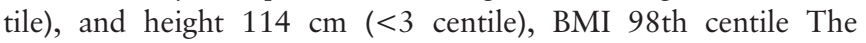
skin was dry and pale. The test showed TSH $-820 \mathrm{mIU} / \mathrm{L}$, T4f - $1.51 \mathrm{mIU} / \mathrm{L}$, and elevated antithyroperoxidase antibodies level. The US of the thyroid gland showed the reduced of the echogenic structure and multiple heterogeneous structures, confirming The Primary Hypothyroidism due to Autoimmune thyroiditis. MRI head reported The Pituitary Hyperplasia/Pituitary Adenoma secondary to The Primary Hypothyroidism. She was commenced on levothyroxine $50 \mu \mathrm{g} / \mathrm{kg}$ once daily. The repeated test after 10 days of treatment showed TSH 448.4 $\mathrm{mIU} / \mathrm{L}$ T3 $2.49 \mathrm{mIU} / \mathrm{L}$, T4 $7.15 \mathrm{mIU} / \mathrm{L}$. She was discharged home on levothyroxine and regular monitoring of TSH.
Conclusion Thyroid hormone is a major contributor to postnatal growth and hypothyroidism occurring postnatally can result in profound growth failure and arrest of skeletal maturation. The danger of chronic autoimmune thyroiditis disease lies in the fact that the disease is almost asymptomatic. Pituitary hyperplasia usually developed due to hypothyroidism, that responds well to thyroid hormone replacement therapy.

\section{THE ROLE OF INTERACTION BETWEEN HOSPITALS IN HEALTH SYSTEM}

Ani Asatryan*, E Harutyunyan, L Shahumyan, A Aghajanyan, H Shahumyan, S Khachatryan, I Atoyan, A Sahakyan, G Manukyan, K Esoyan, S Mirabyan, A Sahakyan, M Grigoryan, B Baveyan, V Asoyan, H Apresyan. Yerevan State Medical University

\subsection{6/archdischild-2021-europaediatrics.498}

The aim of our research was to investigate the diseases of 0 18 year old patients admitted to the 'Muratsan' University hospital complex (UHC) Intensive care unit (ICU) and toxicology for the last 7 years, including cases that have been reported by the 'Muratsan' UHC (ICU) from the hospitals in Yerevan and provinces and other departments of 'Muratsan' UHC.

A retrospective study was performed using medical charts up to 7 years(2012-2018) from 'Muratsan' UHC ICU and toxicology. The overall number of patients was 5152, among them 3074 were male and 2078 were female. This study included cases that have been reported by the 'Muratsan' UHC ICU and toxicology from the hospitals in Yerevan and provinces and other departments of 'Muratsan' UHC. The overall number of patients who were referred from the hospitals of Yerevan is $62(1.2 \%)$. 20(32.3\%)-Acute respiratory infection(ARI), 17(27.4\%)-Acute Intoxications(AI), 18(29\%)-Acute Drug Intoxications(ADI) and 1(1.6\%)-allergic disorders, 6 (9.7\%)-other disorders*. The referred patients were divided into 4 age groups $(0-1,1-7,7-14,14-18)$, the first group-13, the second-37, the third-2 and the fourth- 10 cases. 25 (40.3\%)-moderate, 35(56.5\%)-severe and 2(3.2\%)-extremely severe cases.

681 patients $(13.2 \%)$ were referred from regional hospitals, 38(5.6\%) patients had ARI, 400(58.7\%)-AI, 174(25.6\%)-ADI and 22(3.2\%)-allergic disorders, 47(6.9\%)-other disorders. The first age group included-72, the second-500, the third-79 and the fourth-30 cases. 334(49\%)-moderate, 345(50.7\%)-severe cases, 2(0.3\%)-extremely severe cases.

The number of internal directions was 157(3\%): 92 (58.6\%)-ARI, 7(4.5\%)-AI, 6(3.8\%)-ADI and 4(2.5\%)-allergic disorders, 48(30.6\%)-other disorders. The first age group included-26, the second-116, the third-10 and the fourth-5 cases. $11(7 \%)$ were moderate, $139(88.5 \%)$-severe and $7(4.5 \%)$ extremely severe cases.

*Diabetes mellitus, epilepsy, cancer, hematological disorders, etc.

We came to the conclusion that most cases of severe intoxication among children aged 1 to 7 years were transferred from different hospitals in Yerevan, regions and other departments of 'Muratsan' UHC. From this we can conclude that as a result of the organized work of the primary rings, patients are sent to the necessary specialized department. 